\title{
Different distributions of operative diagnoses for Achilles tendon overuse injuries in Italian and Finnish athletes
}

\author{
Kristian Johansson ${ }^{1}$ \\ Lasse Lempainen ${ }^{2}$ \\ Janne Sarimo ${ }^{2}$ \\ Tiina Laitala-Leinonen ${ }^{3}$ \\ Sakari Orava ${ }^{2}$ \\ 1 Department of Paavo Nurmi Center, Turun \\ Yliopisto, Turku, Finland \\ 2 Hospital Neo, Turku, Finland \\ 3 Skeletal Biology Consortium, Department of Cell \\ Biology and Anatomy, University of Turku, Turku, \\ Finland
}

Corresponding author:

Kristian Johansson

Department of Paavo Nurmi Center, Turun Yliopisto

Kiinamyllynkatu 10

20520 Turku, Finland

E-mail: kristian.johansson@utu.fi

\section{Summary}

Background: the origin of chronic Achilles tendinopathy (AT) is currently unclear and epidemiological factors, such as ethnicity, may be associated.

Methods: intraoperative findings from the treatment of $\mathbf{8 6 5}$ Finnish and $\mathbf{1 5 6}$ Italian athletic patients with chronic Achilles tendon related pain were evaluated, retrospectively. The mean age was 34 years (range, 18 to 65 years) in the Finnish and 29 years (range, 17-63 years) in the Italian patients. In total, 786 patients were males and 226 females of which 84 and $87 \%$ Finnish, respectively. Data were collected, retrospectively from patient records. The differences in the frequencies of operative findings were assessed for statistical significance.

Results: retrocalcaneal bursitis, partial tear and chronic paratenonitis were the most prevalent findings in patients with chronic AT undergoing surgery. Tendinosis and chronic paratenonitis were significantly $(p=0.011)$ more common in Finnish athletes. Italian patients exhibited significantly $(p<0.001)$ more insertional calcific tendinopathy (heel spurs) and prominent posterosuperior calcaneal corners (Haglund's heel).

Conclusion: ethnicity appears to be associated with specific characteristics of overuse-related Achilles tendon pathology. This is an issue that should be considered in the planning of genetic research on AT.

KEY WORDS: Achilles tendon, overuse injury, surgery, tendinopathy.

\section{Introduction}

The etiology of Achilles tendon overuse related injuries remains in dispute. Neither has a definitive, curative treatment yet been discovered. Achilles tendinopathy (AT) is a serious concern because of its chronicity and resistance to treatment. By definition, AT is recognized by local swelling and tenderness and impaired function and is therefore a clinical diagnosis ${ }^{1}$. By anatomical location, AT is closely related to retrocalcaneal and superficial calcaneal bursitis ${ }^{2}$. Beyond the clinical diagnosis of tendinopathy, several macro- and microscopical pathologies exist, such as tendinosis, chronic paratendinopathy and calcific tendinopathy ${ }^{2,3}$. A clinical distinction is not always evident among these closely related tissues, and even more rare conditions causing chronic Achilles tendon pain exist. Surgical treatment is sometimes opted in cases unresponsive to non-operative treatment. The basic pathology leading to tendon degeneration and pain lies possibly in altered biochemical and neuronal signaling ${ }^{2,4-6}$. Risk factors for predisposition of tendinopathy are unclear, including inconclusive intrinsic and extrinsic factors ${ }^{7,8}$. Although physical activity and related overuse is often associated with tendinopathy, it is not solely a disease of the athletically active $e^{9,10}$. Some epidemiological theories have been put forth, such as age and gender, although differences among ethnicities are unclear ${ }^{11}$.

The clinical diagnosis of AT is frequently used in literature, while several macroscopical pathologies are observed in surgery. Currently the distributions of Achilles tendon pathologies in surgery are not well understood due to limited reports with large series. Genetic risk factors for AT have been discovered, most notably in the COL5A1 gene ${ }^{12}$. Existing intrinsic predispositions for AT might also be expressed between different ethnicities.

\section{Materials and methods}

In this retrospective observational study, 1012 consecutive operations for Achilles tendinopathy in pro- 
fessional and recreational athletes from Finland and Italy were assessed retrospectively from medical charts. All the operations were done by a single surgeon during the years 1990-2004 in Finland and Italy. Inclusion criteria were patients with Achilles tendinopathy and exclusion criteria were total tendon rupture and patients with rheumatic history or findings. The Finnish and Italian patients were native to their respective home country. All patients presented initially pain in the unilateral hind foot at the surgeon's offices either in Finland or in Italy. Clinical evaluation, along with plain radiography, with or without MRI, and appropriate treatment measures were chosen individually according to the clinical diagnosis. Conservative treatment was given prior to surgical treatment either at previous clinics or at the surgeon's office. Conservative treatment included, but was not limited to: eccentric calf muscle exercises; muscle stretching; NSAIDs; extracorporeal shock wave treatment (ESWT); local corticosteroid injections; shoe modifications and a modified training program.

In persistent cases unresponsive to treatment, with symptoms lasting for a minimum of 6 months $(n=1012)$, surgery was opted for. In surgery, the problem was explored and the preliminary clinical diagnosis was either confirmed or changed accordingly to findings. Data for postsurgical diagnosis as well as age, sex, and type of sports activity were collected from medical charts. Data was not however individualised.

Diagnostic classification was based on the same criteria in all patients and was done by macroscopic intraoperative observation ${ }^{13,14}$. A prominent postero-superior calcaneal tuberosity and Achilles tendon insertional calcification was diagnosed by lateral radiography as well as macroscopic observation. A Haglund's heel was diagnosed if a heel had a pointed lateral angle of the postero-superior calcaneal corner of less than $90^{\circ}$, with or without retrocalcaneal bursitis and ventral Achilles tendon attrition. In those cases when several pathologies were observed, the main pathology was chosen based on a subjective evaluation of its relative prominence or severity.

The distribution of the operative diagnoses were tabled and analysed by binary logistic regression analysis with IBM SPSS statistics version 21, New York, US. Significant differences had a p-value of less than 0.05 . A power calculation of sample size was not done because of the retrospective design of this study. The confidence intervals were set at $95 \%$ probability. The study included 856 Finnish and 156 Italian patients, of which 659 (77\%) and 127 (81\%) were male respectively. The mean age was 34 years (range: 18 to 65 years) in the Finnish group and 29 years (range: 17 to 63 years) in the Italian group. Endurance sports were the main discipline in $80 \%$ of the Finnish athletes, and in $72 \%$ of the Italian athletes. Patient characteristics are presented in the Table 1. The study meets the ethical standards of the Journal ${ }^{15}$.

\section{Results}

Overall distribution of diagnoses is presented in the Figure 1, where the numbers in the Figure represent the percentage of that diagnosis in each nationality. Retrocalcaneal bursitis, partial tear of the tendon and chronic paratenonitis were the three most frequently observed pathologies. Retrocalcaneal bursitis was the most common finding in both groups. The two second most common findings in the Finnish group were partial tear and chronic paratenonitis while in the Italian group partial tear and Haglund's heel. The Finnish athletes had an odds ratio of $2.76[\mathrm{Cl}=1.32$ 5.77] for tendinosis $(p=0.007)$ and $2.03[\mathrm{Cl}=1.26$ 3.27] for chronic paratenonitis $(p=0.004)$, compared to their Italian peers. In addition, the risk ratio for insertional calcific tendinopathy and Haglund's heel was .21 [.12 - .39] $(p=<0.001)$ and .28 [.17 - .45]

Table 1. Surgical diagnosis and group characteristics in Finnish and Italian athletes.

\begin{tabular}{|c|c|c|c|c|}
\hline Nationality & Finnish & Italian & & \\
\hline Gender $\mathrm{f} / \mathrm{m}$ & $197 / 659$ & $29 / 127$ & & \\
\hline Mean Age (range) & 34 y $(18-65 y)$ & 29 y $(17-63 y)$ & & \\
\hline Diagnosis & $\mathrm{n}(\%)$ & $\mathrm{n}(\%)$ & OR $[95 \% \mathrm{Cl}]$ & $p$-value \\
\hline Retrocalcaneal bursitis & $231(27)$ & $44(28)$ & $.94[.64-1.38]$ & .753 \\
\hline Partial tear & $214(25)$ & $30(19)$ & $1.40[.91-2.15]$ & .123 \\
\hline Chronic paratenonitis & $214(25)$ & $22(14)$ & $2.03[1.26-3.27]$ & .004 \\
\hline Haglund's heel & $51(6)$ & $29(19)$ & $.28[.17-.45]$ & $<.001$ \\
\hline Tendinosis & $111(13)$ & $8(5)$ & $2.76[1.32-5.77]$ & .007 \\
\hline Insertional calcific AT (heel spur) & $26(3)$ & $20(13)$ & $.21[.12-.39]$ & $<.001$ \\
\hline Anomalous soleus & $9(1)$ & $3(2)$ & $.54[0.15-2.02]$ & .362 \\
\hline Total & $856(100)$ & $156(100)$ & & \\
\hline
\end{tabular}




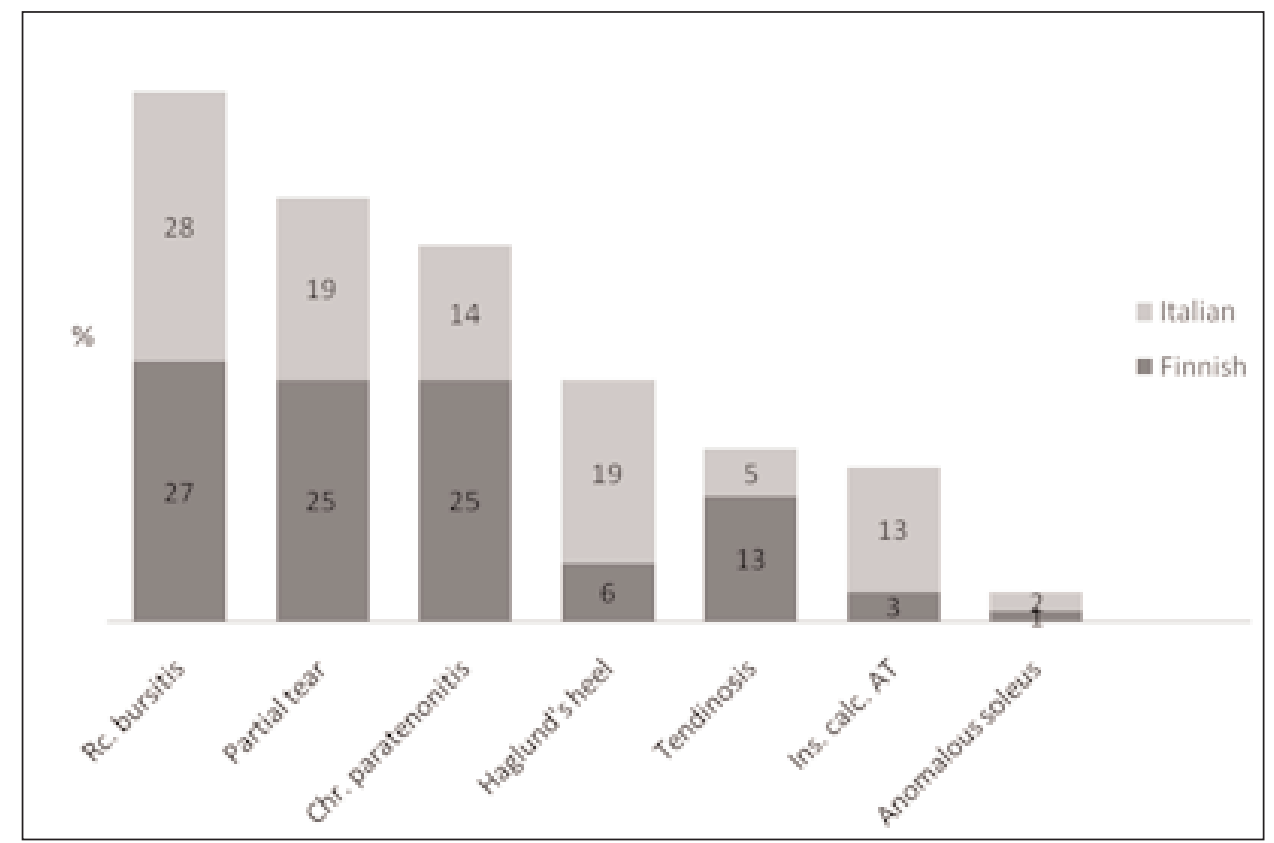

Figure 1. Proportional distribution of main surgical diagnoses in Finnish and Italian athletes. The numbers represent the percentage of that diagnosis in each nationality.

$(p=<0.001)$ respectively for the Finnish patients. Retrocalcaneal bursitis, partial tear and anomalous soleus muscle did not, however, differ significantly in prevalence.

\section{Discussion}

This study is the first to systematically compare surgical findings in Achilles tendinopathy between two different ethnic populations. We observed a significantly greater proportion of surgically confirmed insertional calcific AT and Haglund's heels amongst Italian athletes compared to Finnish athletes suffering from chronic Achilles tendinopathy. Tendinosis and chronic paratenonitis were also significantly more prevalent in Finnish patients, but the difference appeared slightly less evident due to the broad confidence interval. When the material was gathered, this study was not yet conceived which causes several limitations to be considered. Firstly, we cannot ascertain if there was a selection bias in one or both ethnic groups and this should be studied by comparing results from other orthopedic clinics. Other ethnicities were not included because we only had large groups of Italian and Finnish patients. Secondly, case-control matches were not available in regard of same amount of physical exercise, gender, age, event and evenly powered groups. Due to non-individualised data of the study subjects, we could not perform more accurate statistical analysis where the correlation of e.g. age and gender to the distribution of the diagnosis could have been taken to account. A previous study by Longo et al., however, found no influence of gender, age and impact profile and other variables on the prevalence of clinical tendinopathy in track and field athletes ${ }^{16}$. The diagnosis for tendinosis and paratendinopathy is confirmed after histopathological confirmation ${ }^{1}$. In this study, the aim was to evaluate macroscopic findings only. Tendinosis is not always observable by inspection and histopathological samples may have revealed clinically non manifested tendinosis ${ }^{17}$. Partial rupture, for example, should be considered a continuum of tendinosis, but appreciated for its potential significance in clinical practice ${ }^{18}$. Another issue is the presence of comorbidities in the diseased tendon and retrocalcaneal space, which were not addressed in the Table of surgical findings. Observed pathologies were not always limited to the main surgical findings listed in the Table, as macroscopically assessed mild degrees of attrition (partial tear) on the Achilles tendon, paratenonitis and retrocalcaneal bursitis may have been present. Of all the patients with retrocalcaneal bursitis, a Haglund's heel by previous definitions was observed in $6 \%$ of Finnish and $19 \%$ of Italian patients. Insertional calcific spurs in tendons can develop without inflammation or microtrauma correlated with physical exercise and is found in asymptomatic patients ${ }^{19-22}$. Histology shows intracalcific stem cell activity along with fast remodellation and chondral metaplasia including abnormal quantities of collagen type II and III in adjunct tendon ${ }^{3,23}$. The anomalous soleus has been described earlier ${ }^{24}$ and is radiologically recognized correctly by MRI.

Generalization of Achilles tendon overuse injuries in- 
to tendinopathy could lead to misconceptions. An excellent guideline for terminology and clinicopathological definitions was done by Van Dijk et al. ${ }^{2}$. In nonoperative studies, it is difficult to make a conclusion of the true lesion by clinical and radiological observations alone because the diagnosis can appear more complex or incorrect after surgical exploration. On the other hand, the various pathologies in and around the Achilles tendon may respond differently to non-surgical and surgical treatment, confounding the interpretation of results in clinical trials if not taken into account. Also, tendons from different locations may develop tendinopathy in different manners ${ }^{25}$.

Explanations for differences in AT surgical diagnoses between the Italian and Finns could be due to environmental effects. Factors that might be causing more calcification in Italian patients could be derived from potentially calcium-rich water from the Dolomites in the north of the country, but this is not verified. Other calcium related factors include the sun dependent, skin-derived D-vitamin activity that is low during the long periods of relatively sparse sunlight in Finland.

Surface characteristics can also be an independent factor, since the climate is very different in Finland and Italy during the long winter period, when Nordic countries are mainly dependent of indoor training grounds. There is not an established understanding, however, on the relation of surface characteristics on tendinopathy 26 .

Basic research on the pathomechanisms of tendinopathy is occurring simultaneously as the efficacy of novel treatments is evaluated. There is a consensus, that the reasons for tendon pain and degeneration must be elucidated before the tendinopathy problem can be solved. New intrinsic factors have been discovered through genomic research, such as the COL5A1 gene, and extrinsic factors are neither well understood although correlations to tendinopathy exist, such as tendon load ${ }^{12,27}$. This present study promotes two approaches to elucidate AT pathology. Firstly, ethnic differences should be considered in the development of Achilles tendon related overuse injuries, and this should, for example, encourage ethnic comparison of collagen coding gene sequences. Secondly, different abnormalities can be found in and around the tendon causing achillodynia and thus researchers studying tendinopathy should be aware of these when planning trials and performing studies in the search of the causa prima non causata.

\section{Conflict of interests}

The Authors declare that they have no conflict of interests regarding the publication of this paper.

\section{References}

1. Maffulli N, Khan KM, Puddu G. Overuse tendon conditions: time to change a confusing terminology. Arthroscopy. 1998;14(8):840-843.
2. Van Dijk CN, van Sterkenburg MN, Wiegerinck JI, Karlsson J, Maffulli N. Terminology for Achilles tendon related disorders. Knee Surg Sports Traumatol Arthrosc. 2011;19(5):835-841.

3. Johansson KJJ, Sarimo JJ, Lempainen LL, Laitala-Leinonen T, Orava SY. Calcific spurs at the insertion of the Achilles tendon: a clinical and histological study. Muscles Ligaments Tendons J. 2012;2(4):273-277.

4. Benjamin M, Toumi H, Ralphs JR, Bydder G, Best TM, Milz S. Where tendons and ligaments meet bone: attachment sites ('entheses') in relation to exercise and/or mechanical load. J Anat.2006;208(4):471-490.

5. Lui PPY, Maffulli N, Rolf C, Smith RKW. What are the validated animal models for tendinopathy? Scand J Med Sci Sports. 2011;21(1):3-17.

6. Van Sterkenburg MN, van Dijk CN. Mid-portion Achilles tendinopathy: why painful? An evidence-based philosophy. Knee Surg Sports Traumatol Arthrosc. 2011;19(8):1367-1375.

7. Almekinders LC, Temple JD. Etiology, diagnosis, and treatment of tendonitis: an analysis of the literature. Med Sci Sports Exerc. 1998;30(8):1183-1190.

8. Rees JD, Maffulli N, Cook J. Management of tendinopathy. Am J Sports Med. 2009 [cited 2014 Feb 19];37(9):1855-1867.

9. Rolf C, Movin T. Etiology, histopathology, and outcome of surgery in achillodynia. Foot Ankle Int. 1997;18(9):565-569.

10. Ames PRJ, Longo UG, Denaro V, Maffulli N. Achilles tendon problems: not just an orthopaedic issue. Disabil Rehabil. 2008;30(20-22):1646-1650.

11. Kannus P. Etiology and pathophysiology of chronic tendon disorders in sports. Scand J Med Sci Sports. 1997;7(2):78-85.

12. Abrahams $Y$, Laguette M-J, Prince S, Collins M. Polymorphisms within the COL5A1 3'-UTR that alters mRNA structure and the MIR608 gene are associated with Achilles tendinopathy. Ann Hum Genet. 2013;77(3): 204-214.

13. Puddu G, Ippolito E, Postacchini F. A classification of Achilles tendon disease. Am J Sports Med. 1976;4(4):145-150.

14. Williams JGP. A color atlas of injury in sports. London: Wolfe Medical Publ Ltd. 1980.

15. Padulo J, Oliva F, Frizziero A, Maffulli N. Muscle, Ligaments and Tendons Journal. Basic principles and recommendations in clinical and field science research. MLTJ. 2013;3(4):250252.

16. Longo UG, Rittweger J, Garau G, et al. No influence of age, gender, weight, height, and impact profile in achilles tendinopathy in masters track and field athletes. Am J Sports Med. 2009;37(7):1400-1405.

17. Aström M, Rausing A. Chronic Achilles tendinopathy. A survey of surgical and histopathologic findings. Clin Orthop Relat Res. 1995;(316):151-164.

18. Aström M. Partial rupture in chronic achilles tendinopathy. A retrospective analysis of 342 cases. Acta Orthop Scand. 1998;69(4):404-407.

19. Krahl H, Pieper HG, Quack G. Die Knochenhypertrophie als Trainingseffekt. Orthopade. 1995;24(5):441-445.

20. Benjamin M, Rufai A, Ralphs JR. The mechanism of formation of bony spurs (enthesophytes) in the achilles tendon. Arthritis Rheum. 2000;43(3):576-583.

21. Tyrdal S, Finnanger AM. Osseous manifestations of "handball goalie"s elbow'. Scand J Med Sci Sports. 1999;9(2):92-97.

22. Bassiouni M. Incidence of calcaneal spurs in osteo-arthrosis and rheumatoid arthritis, and in control patients. Ann Rheum Dis. 1965;24(5):490-493.

23. Maffulli N, Reaper J, Ewen SWB, Waterston SW, Barrass V. Chondral metaplasia in calcific insertional tendinopathy of the Achilles tendon. Clin J Sport Med 2006;16(4):329-334.

24. Christodoulou A, Terzidis I, Natsis K, Gigis I, Pournaras J. Soleus accessorius, an anomalous muscle in a young athlete: case report and analysis of the literature. $\mathrm{Br} \mathrm{J}$ Sports Med. 2004;38(6):e38. 
2004;38(6):e38.

25. Giombini A, Dragoni S, Di Cesare A, Di Cesare M, Del Buono A, Maffulli N. Asymptomatic Achilles, patellar, and quadriceps tendinopathy: a longitudinal clinical and ultrasonographic study in elite fencers. Scand J Med Sci Sports. 2013;23(3):
311-316.

26. Nigg BM. The role of impact forces and foot pronation: a new paradigm. Clin J Sport Med 2001;11(1):2-9.

27. Cook J, Khan K. Etiology of tendinopathy. In: S. Woo, P. Renstrom SA, editor. London: Wiley-Blackwell. 2007;10-28. 